\title{
Os desafios do financiamento do enfrentamento à COVID-19 no SUS dentro do pacto federativo
}

\author{
Gustavo Andrey de Almeida Lopes Fernandes 1 \\ Blenda Leite Saturnino Pereira ${ }^{2}$ \\ 1 Fundação Getulio Vargas / Escola de Administração de Empresas de São Paulo, São Paulo / SP - Brasil \\ 2 Universidade de São Paulo / Faculdade de Saúde Pública, São Paulo / SP - Brasil
}

\begin{abstract}
O aprimoramento das regras de financiamento do Sistema Único de Saúde (SUS) em estados e municípios é um dos grandes desafios da saúde pública no Brasil. O objetivo deste artigo é contribuir para este amplo debate, analisando a resposta governamental ao desafio de combate à COVID-19, sob o prisma do financiamento público dos serviços de saúde dos governos subnacionais brasileiros. Uma abordagem quali-quantitativa é adotada, mesclando-se análise documental e análise de regressão. Resultados mostram que não houve mudança substantiva nos critérios de repasse, pouco sensíveis a fatores epidemiológicos. Ajustes nas normas de aplicação foram realizados para dar maior agilidade aos gastos. Tamanho populacional, produção de riquezas locais e número de leitos de internação parecem ser os principais fatores que definem a distribuição dos recursos. O desenho de financiamento do combate à COVID-19, assim como o volume de recursos parecem ser insuficientes frente à dimensão da crise.
\end{abstract}

Palavras-Chave: COVID-19; saúde; administração pública; finanças públicas; federalismo.

\section{Los desafíos de la financiación del enfrentamiento a la COVID-19 en el SUS dentro del pacto federativo}

La mejora de las normas de financiación del Sistema Único de Salud (SUS) en los estados y municipios es uno de los principales desafíos de salud pública en Brasil. El propósito de este artículo es contribuir a este amplio debate, analizando la respuesta del gobierno al desafío de combatir COVID-19, bajo el prisma de la financiación pública para los servicios de salud de los gobiernos subnacionales brasileños. Se adopta un enfoque cualitativo cuantitativo, que combina el análisis documental y el análisis de regresión. Los resultados muestran que no hubo cambios sustanciales en los criterios de transferencia, poco sensibles a los factores epidemiológicos. Se hicieron ajustes a las reglas de aplicación para acelerar el gasto. El tamaño de la población, la producción de riqueza local y el número de camas de hospital parecen ser los principales factores que definen la distribución de los recursos. El diseño de financiamiento para combatir COVID-19, así como el volumen de recursos, parece ser insuficiente en vista de la magnitud de la crisis.

Palabras clave: COVID-19; salud; administración pública; finanzas públicas; federalismo.

\section{The challenges of funding the Brazilian health system in fighting the COVID-19 pandemic in the context of the federative pact}

The improvement of rules to fund the Brazilian health system (SUS) in states and municipalities is one of the major public health challenges in Brazil. The purpose of this article is to contribute to this broad debate, analyzing the government's response to the challenge of combating COVID-19, from the perspective of public financing of health services of Brazilian subnational governments. A qualitative and quantitative approach is adopted, combining documentary analysis and regression analysis. The results show that there was no substantive change in the criteria for transfers, which are not sensitive to epidemiological factors. Adjustments to the application rules were made to speed up spending. Population size, production of local wealth, and the number of hospital beds are the main factors that define the distribution of resources. The funding design for combating COVID-19, as well as the volume of resources, are insufficient in view of the scale of the crisis.

Keywords: COVID-19; health; public administration; public finance; federalism. 


\section{INTRODUÇÃO}

O combate aos efeitos da COVID-19 tornou-se uma urgência global (Wang, Horby, Hayden, \& Gao, 2020). Desde o início do surto na China, diversos países foram acometidos, com enorme impacto na economia e, sobretudo, em seus sistemas de saúde. Estas primeiras experiências têm sido de vital importância para os países acometidos em seguida; entre eles, o Brasil.

Legido-Quigley et al. (2020) enumeram os aprendizados com o caso da Espanha. Em primeiro lugar, recursos financeiros adicionais são necessários para apoiar governos subnacionais. Em segundo lugar, o subfinanciamento em saúde prejudica sua capacidade tanto nos aspectos humanos como materiais. Boa coordenação federativa é fundamental para o enfrentamento eficaz, o que também foi observado no caso da Itália (Anderson, Heesterbeek, Klinkenberg, \& Hollingsworth 2020).

Em virtude da doença, são necessários ajustes imediatos do sistema de saúde tendo em vista o incremento da oferta de determinados serviços (Grasselli, Pesenti, \& Cecconi, 2020). Em influente estudo do Imperial College, Walker et al. (2020) indicam um cenário de colapso dos sistemas de saúde em virtude do pico de demanda, levando ao extremo o número de mortes. No Brasil, Araújo, e Morais (2020), com dados do Ceará, já apresentam sinais nesta direção. Para Walker et al. (2020), medidas de mitigação, tais como distanciamento social, são extremamente necessárias, gerando melhores condições para o sistema de saúde.

Diante disso, uma resposta imediata é o aumento dos gastos em saúde (Legido-Quigley et al., 2020). No entanto, neste aspecto, o cenário brasileiro não é favorável. Vieira e Santos (2018), analisando dados da execução do Ministério da Saúde (MS) entre 2002 a 2015, constatam que os limites autorizados de pagamento foram insuficientes para fazer frente aos gastos em cada exercício, ilustrando a insuficiência de recursos. Marques (2017) mostra que o subfinanciamento do SUS no Brasil é estrutural, não havendo forte apoio político para a destinação de maiores recursos ao sistema. Com dados mais recentes, Pereira e Faleiros (2019) mostram que renúncias fiscais nos últimos anos, além da própria crise econômica, vêm reduzindo os montantes disponíveis para o SUS.

Assim, a despeito da forte restrição de receitas no nível federal, repasses são justamente a principal fonte de recursos para a execução dos serviços de saúde no SUS (Leite, Lima, \& Vasconcelos, 2012; Santos, 2018; Lima \& Andrade, 2009). No entanto, as regras utilizadas têm intensificado a desigualdade no sistema, além de não guardar necessariamente conexão com fatores epidemiológicos ou sociais. Para Lima (2007), por exemplo, falta planejamento das regras que definem a distribuição dos recursos para o financiamento do SUS. Neste aspecto, a pandemia impõe uma série de desafios ao sistema em virtude das características da doença.

Propõe-se, neste trabalho, investigar a compatibilidade das regras de financiamento adotadas com as idiossincrasias da COVID-19. Trata-se de uma contribuição importante para os estudos do tema, considerando-se a possiblidade de uma nova pandemia, ou surtos epidêmicos em diversas regiões do país, sendo fundamental o aperfeiçoamento das regras de financiamento do SUS na formulação de políticas públicas de saúde. Por conseguinte, o objetivo deste artigo é analisar a resposta governamental da União ao desafio de combate à COVID-19, mediante o financiamento público dos serviços de saúde dos governos subnacionais brasileiros, que administram as principais estruturas de resposta à enfermidade.

Este trabalho é composto por sete seções. Segue-se a esta introdução, uma breve revisão da literatura sobre financiamento do SUS, culminando na hipótese de pesquisa. Na seção seguinte, 
é apresentada a metodologia utilizada. Na quarta seção, são analisadas as regras de aplicação, assim como as recentes mudanças. Em seguida, discutem-se a origem dos recursos e, depois, o processo de alocação no nível subnacional. Na sexta seção - seguida da conclusão -, o perfil dos repasses é analisado, indicando-se na prática o quadro geral do financiamento do enfrentamento à pandemia.

\section{O PROBLEMA DO FINANCIAMENTO DOS SERVIÇOS DE SAÚDE}

O financiamento das ações e serviços públicos de saúde, por força constitucional, é de responsabilidade das três esferas de gestão do SUS. No entanto, desde sua institucionalização o financiamento deste sistema sempre foi uma pauta de disputa intensa. Para Mendes (2014), concorrem com a implementação da saúde como direito social a tensão decorrente do discurso de contenção de gastos e a asfixia de recursos.

Mendes e Marques (2009) verificam que entre os anos 1990 e 2000 não houve aumento relevante de financiamento do setor; pelo contrário, ocorreram diversas tentativas de desvinculação. Scatena, Viana, e Tanaka (2009), analisando registros municipais, apontam um crescente descompasso entre receitas e despesas, com forte majoração dos gastos.

Mais recentemente, examinando dados de 2008-2009 e de 2015-2016, Contarato, Lima, e Leal (2019) observam que houve crescimento per capita dos gastos, a despeito da crise econômica, sugerindo a existência de um efeito protetor da vinculação. De todo modo, a recorrente redução do aporte do governo federal, especialmente após a Emenda Constitucional n. 95 (2016), acabou por pressionar os orçamentos dos demais entes, gerando a necessidade de fontes alternativas de receita (Funcia, 2019; Pereira, Oliveira, \& Faleiros, 2019). No período, contudo, estudos evidenciaram a perda da capacidade da oferta de recursos, como mostra O’Dwyer, Graever, Britto, Menezes, e Konder (2019) em um estudo com municípios fluminenses.

Neste contexto, repasses federais têm sido fundamentais à execução dos serviços de saúde no âmbito do SUS, dada a fragilidade das fontes de recursos estaduais e municipais (Lima \& Andrade, 2009; Leite et al., 2012; Santos, 2018). Vazquez (2011) aponta, ainda, para um papel indutor dos gastos locais por parte das transferências condicionais federais. Para Lima (2007), contudo, a falta de um planejamento integrado entre os critérios que regem a redistribuição dos recursos para o financiamento do SUS acaba por reforçar os padrões de assimetria existentes. Piola, França e Nunes (2016) mostram que, mesmo em períodos de crescimento de receita em todos os níveis, como visto no período de 2000 a 2010, as desigualdades regionais, em termos de gasto per capita, praticamente não se modificaram.

Transferências originadas de emendas parlamentares também têm sido uma fonte alternativa de recursos. Porém, Baptista et al. (2012), analisando recursos de emendas de 1997 a 2006, apontam que os critérios seguem muito mais uma lógica política do que um planejamento dos serviços de saúde. Nesse sentido, Santos (2018) aponta a debilidade da ação do próprio MS na melhoria da coordenação regional do SUS pelos estados.

Battesini, Andrade, e Seta (2017), analisando a distribuição de recursos federais da vigilância sanitária de 2005 a 2012, indicam que a adoção de critérios per capita unificados para todo o país levou ao aumento da iniquidade entre os territórios. A reforma dos critérios, contudo, não parece ser tarefa simples. Cabreira, Ritter, Aguiar, e Celeste (2018), com dados de municípios do Rio Grande do 
Sul, indicam que os gasto com saúde parecem estar mais vinculados às políticas federais de indução do que aos fatores associados com a demanda em saúde, como o perfil demográfico e epidemiológico. Resultados similares para municípios baianos são encontrados por Teles, Coelho, e Ferreira (2016). Harzheim et al. (2020), por sua vez, sugerem um sistema de distribuição de repasses baseado em indicadores de vulnerabilidade socioeconômica, de aspectos demográficos, além do pagamento por desempenho por meio de indicadores, endossando as críticas aos critérios atuais.

O problema da fragilidade do financiamento dos serviços prestados pelo SUS é recorrente. No caso de uma pandemia causada por uma doença nova, ainda carente de grandes estudos, o desafio é ainda maior. Segundo Weiss e Murdoch (2020), cerca de 19\% das pessoas com COVID-19 necessitam de hospitalização, enquanto a taxa de mortalidade de casos de infecção é de aproximadamente 2,2\% (Chan et al., 2020). Comunidades densas correm um risco próprio, uma vez que o vírus pode ser transmitido por meio de aerossóis, fica comprometida uma das ferramentas mais importantes para combater a pandemia, o distanciamento social. Para mitigar as consequências da pandemia, são necessários ajustes imediatos no sistema de saúde que aumentem a oferta de serviço de modo a atender à demanda excepcional da infecção pelo vírus (Anderson et al., 2020; Grasselli et al., 2020). Em um estudo influente no Imperial College, Walker et al. (2020) indicam um cenário de colapso dos sistemas de saúde devido ao pico de demanda, levando ao extremo o número de mortes.

Por conseguinte, conforme o panorama de iniquidade da repartição de recursos do SUS e as características da COVID-19, chega-se à hipótese desta pesquisa.

Hipótese: o modelo de financiamento do SUS dentro do pacto federativo brasileiro foi modificado em razão das necessidades de enfrentamento da COVID-19.

Em outras palavras, busca-se inferir se o volume de recursos e as regras empregadas no custeio das políticas de combate à COVID-19 foram influenciados diretamente pelas características da pandemia, dentro da lógica de financiamento do SUS no pacto federativo brasileiro.

Como destacado nesta breve revisão de literatura, critérios de alocação de recursos para transferências com base em número de habitantes ou de usuários dos serviços de saúde prestados são frequentes no SUS. Não obstante, as características da COVID-19, assim como o contágio heterogêneo em um país de tamanho continental como o Brasil impõem uma série de desafios de ordem prática. $\mathrm{Na}$ Espanha, por exemplo, o sistema nacional de saúde é caracterizado por forte descentralização, com grande atuação das comunidades autônomas (Pereira et al., 2019). Entretanto, o enfrentamento da pandemia impôs uma centralização do sistema de saúde sem precedentes no país, modificando, ao menos temporariamente e de modo profundo, a sua dinâmica de funcionamento (Legido-Quigley et al., 2020).

\section{MÉTODOS}

Para responder a questão levantada por esta pesquisa, sobre a compatibilidade entre as regras de financiamento adotadas pelo SUS e as idiossincrasias da COVID-19, emprega-se uma estratégia de métodos mistos (McNabb, 2002). Em uma primeira etapa, é realizada uma ampla análise documental sobre a legislação que define as regras de financiamento do combate à COVID-19. O Esquema 1 detalha o processo. 


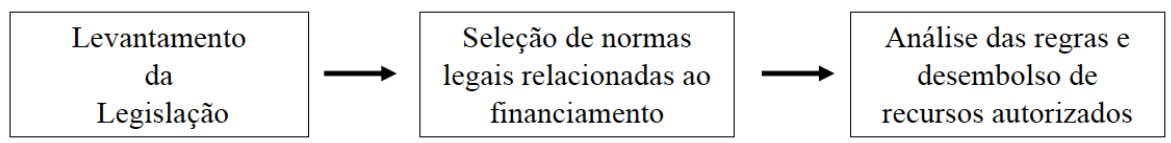

Fonte: Elaborado pelos autores.

Preliminarmente foi realizado um levantamento da legislação envolvida nos sítios eletrônicos do MS e também na Imprensa Oficial da União de 01 de fevereiro até 12 de junho de 2020, abordando o financiamento das políticas de enfrentamento da COVID-19 no nível subnacional. Num segundo passo, foram selecionadas as normas que abrangem especificamente a definição das regras e a autorização dos desembolsos. Encerrando a fase qualitativa, examinaram-se as regras e os recursos desembolsados, o que permitiu desenhar o modelo de financiamento implícito.

$\mathrm{Na}$ segunda etapa da pesquisa, foram analisados os desembolsos efetivamente recebidos pelos municípios, buscando inferir se o modelo de financiamento do SUS, dentro do pacto federativo brasileiro, é consistente em relação às características epidemiológicas da COVID-19. Assim, estimamos uma função linear pelo método dos mínimos quadrados ordinários, com estimadores robustos (Greene, 2002):

$$
\begin{gathered}
\text { Equação } 1 \\
y_{i}=a_{0}+a_{1} X_{i}+\alpha_{2} Z_{i}+a_{3} C_{i}+u_{0}
\end{gathered}
$$

A variável dependente indica o total de repasses recebidos pelo município i. Os dados foram obtidos do portal da transparência do Fundo Nacional da Saúde (FNS) (https://painelms.saude. gov.br/extensions/TEMP_COVID19/TEMP_COVID19.html). Por sua vez, é um vetor de variáveis que descreve o potencial de expansão da doença. Assim, seguindo Feng et al. (2020) e Wang et al. (2020), usou-se a densidade populacional do município, conforme o papel do adensamento no grau de contágio, assim como a distância até a respectiva capital estadual e até Brasília, como proxy para captar o impacto em virtude de o município estar próximo aos principais pontos de entrada do estado afetado pela COVID-19 - dados do Instituto Brasileiro de Geografia e Estatística (IBGE). Supondo que as regras de financiamento sejam consistentes com as características da doença, busca-se inferir se ambos os coeficientes são positivos e estatisticamente significantes.

Já reflete um conjunto de variáveis explicativas que buscam avaliar o potencial de expansão do SUS local para suprir a demanda excessiva decorrente da enfermidade. Com este propósito, utilizam-se o gasto por saúde per capita, o número de leitos de internação e de tratamento intensivo.

Também são incluídas variáveis de controle () que descrevem as condições socioeconômicas dos municípios, como a produção de riquezas no município (PIB), o número de habitantes, se se trata de capital estadual e, por fim, o grau de mortalidade infantil (Araújo, Gonçalves, \& Machado, 2017; Kilsztajn, Rossbach, Câmara, \& Carmo, 2003). Todos os dados foram obtidos do Banco de Dados do Tesouro Nacional (FINBRA), FNS/MS, do Cadastro Nacional de Estabelecimentos de Saúde (CNES) e do IBGE. Destaca-se a confiabilidade dos dados coletados no Fundo Nacional de Saúde, que registram os repasses fundo a fundo e permitem o acompanhamento dos saldos bancários das respectivas contas vinculadas. 


\section{RESULTADOS}

\subsection{Regras básicas de aplicação: mudanças e consequências}

As normas de transferências de valores para governos subnacionais estão regulamentadas na Portaria de Consolidação n. 6 (2017). Os recursos federais são repassados na modalidade fundo a fundo, ou seja, do FNS para os respectivos fundos de saúde de estados e municípios. O sistema de financiamento da saúde pública possuía seis blocos distintos até 2017, tendo sido reduzido a apenas dois blocos: o de custeio das ações e serviços e o de investimento na rede de serviços públicos. A evolução normativa é descrita na Figura 1.

FIGURA 1 LEGISLAÇÃO: REGRAS BÁSICAS DE APLICAÇÃO

Publicação Port. n. 204 - Criação dos Blocos de Financiamento:

Atenção Básica, Atenção de Média

e Alta Complexidade Ambulatorial

e Hospitalar, Vigilância em Saúde,

Assistência Farmacêutica, Gestão do SUS

- 29 de janeiro de 2007
Revogação Port. n. 204 e Publicação Port. n. 3992 - Criação: Bloco de

Custeio das Ações e Serviços

Públicos de Saúde; Bloco de Investimento na Rede de Serviços Públicos de Saúde

- 28 de dezembro de 2017
Alteração Port. n. 3992 e Publicação Port. n. 828 - Blocos passam a ter nova nomeclatura: Bloco de Manutenção das Ações e Serviços Públicos de Saúde , Bloco de Estruturação da Rede de Serviços Públicos de Saúde

- 20 de abril de 2020

Fonte: Elaborada pelos autores com base em Imprensa Nacional.

Esta divisão estabelece que os recursos que compõem cada bloco de financiamento devem ser aplicados estritamente nos itens de despesa pertencentes ao próprio bloco e de acordo com a portaria que originou o repasse. Assim, os entes subnacionais devem organizar-se e atender, para recepção dos incentivos federais, as normas de aplicação definidas pelo ente transferidor, tendo como uma de suas vedações a não autorização de quaisquer remanejamentos de valores entre os blocos; as sobras de valores devem ser mantidas em aplicação financeira de resgate automático até a sua data de utilização.

A ausência de discricionariedade trouxe dificuldades para a gestão dos recursos no enfrentamento da COVID-19, que foram mitigadas apenas com a edição da Portaria n. 828 (2020), em 17 de abril de 2020. Na fase inicial do combate à pandemia, era expressamente vedado o uso de recursos recebidos no bloco de custeio para adequação da estrutura já existente, devendo qualquer modificação necessária ser paga com montantes do bloco de investimento.

A repercussão dos efeitos da ausência de discricionariedade é um fator relevante. De acordo com dados do FNS, foram disponibilizados a estados, Distrito Federal e municípios um total de $\mathrm{R} \$$ 9,570 bilhões para as ações de enfrentamento da COVID-19. Como a integralidade destes valores foi alocada no então bloco de custeio e posteriormente nomeado como Bloco de Manutenção, as possibilidades de gasto com as adaptações do aparelho de saúde ficaram reduzidas. 
A título de ilustração, com os recursos recebidos do governo federal, os governos subnacionais podem adquirir equipamento de proteção individual, como luvas, máscaras. No entanto, por meio dos recursos do Bloco de Manutenção, não é permitida a compra de novas camas hospitalares, de bomba de infusão para uso com os respiradores, nem de monitor cardíaco, eletrocardiógrafos, dentre outros itens, mesmo que necessários. A análise, por exemplo, do portal de dúvidas do Conselho Nacional de Secretarias Municipais de Saúde (Conasems, 2020) indica a recorrência da solicitação de esclarecimentos neste sentido. Portanto apenas após meados de abril, com a edição da referida portaria, tais dificuldades foram mitigadas.

\subsection{Os recursos de combate à COVID-19}

Até meados de junho, sete medidas provisórias - relacionadas no Diagrama 1 - haviam sido publicadas, abrindo crédito extraordinário em favor do MS para enfrentamento da pandemia da COVID-19, totalizando 38,96 bilhões. Parte deste recurso tem como origem o remanejamento interno do próprio orçamento da pasta, dado que, em 2020, o total de recursos disponíveis do ministério é de 172,3 bilhões, um valor $21,95 \%$ superior à dotação inicial (134,7 bilhões) e equivalente a $20,12 \%$ da dotação atualizada do ano anterior.

A análise das medidas provisórias mostra que os créditos extraordinários abertos possuem diversas fontes de recursos. Destaca-se o cancelamento de parte de despesas fixadas em ações orçamentárias destinadas a emendas parlamentares individuais. Neste caso, foram $\mathrm{R} \$ 4,8$ bilhões que anteriormente eram destinados ao Incremento Temporário ao Custeio dos Serviços de Assistência Hospitalar e Ambulatorial (R \$ 1,63 bilhões) e ao Incremento Temporário ao Custeio dos Serviços de Atenção Básica em Saúde (R \$ 3,16 bilhões). Além disso, também foi relevante a anulação de dotações orçamentárias relativas a emendas de bancada estadual, de execução obrigatória de outros órgãos (R $\$ 2,0$ bilhões), e recursos novos originários, em sua maioria, de operações de créditos das fontes vinculadas.

\section{DIAGRAMA 1 EVOLUÇÃO DAS MEDIDAS PROVISÓRIAS PUBLICADAS ATÉ 12 DE JUNHO DE 2020}
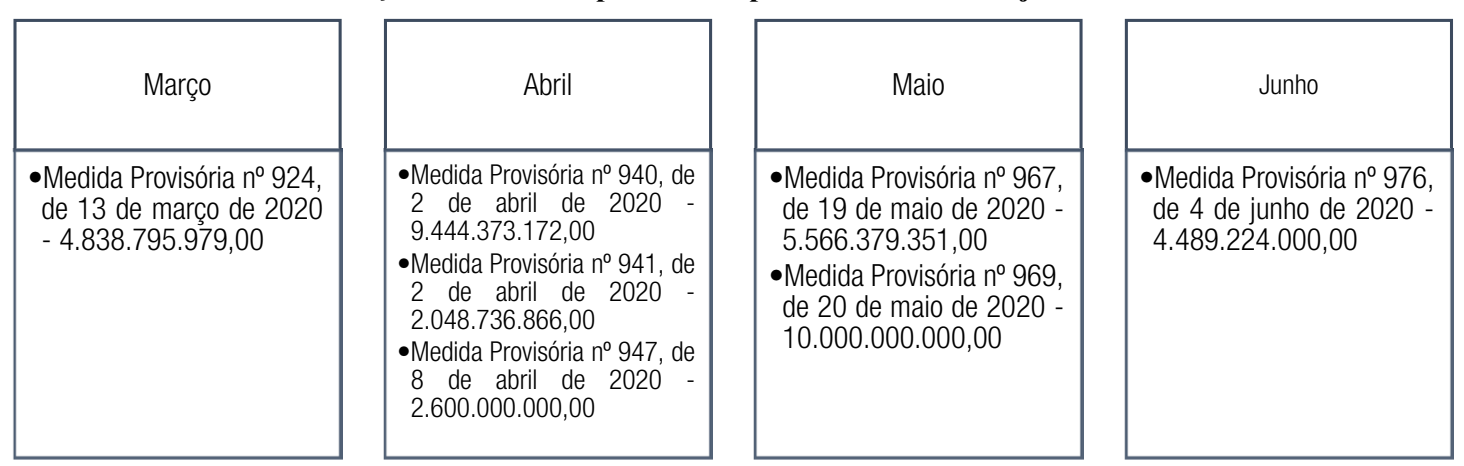

Fonte: Elaborado pelos autores com base em Imprensa Nacional. 
Conforme dados apurados no Sistema Integrado de Planejamento e Orçamento do Governo Federal (SIOP), de 14 de junho de 2020, a maior parcela dos valores destinados ao enfrentamento da COVID-19 divide-se em duas fontes majoritárias: Títulos de Responsabilidade do Tesouro Nacional e Outras Aplicações e Recursos Destinados às Atividades Fins da Seguridade Social.

Por seu turno, a taxa de execução orçamentária tem sido relativamente lenta, especialmente se considerada a estratégia de preparar o sistema de saúde para a curva epidêmica por meio do tempo ganho com o seu achatamento via adoção de medidas de afastamento social (Anderson et al., 2020). Nota-se uma velocidade muito maior de execução na transferência dos recursos dedicados aos municípios e estados, sendo a menor taxa verificada no nível federal.

\section{TABELA 1 VELOCIDADE DE EXECUÇÃO DO COMBATE À COVID-19}

\begin{tabular}{|c|c|c|c|c|c|}
\hline Modalidades de Aplicação & Dotação Atual & $\%$ Dt. AT & Desp. Paga & $\begin{array}{l}\text { \% Desp. } \\
\text { Paga }\end{array}$ & $\begin{array}{c}\text { \%Desp. } \\
\text { atualizada/ } \\
\text { paga }\end{array}$ \\
\hline $\begin{array}{l}\text { Transferências a Estados e ao } \\
\text { Distrito Federal - Fundo a Fundo }\end{array}$ & $9.970 .237 .045,00$ & 25,59 & $3.886 .107 .282,34$ & 34,49 & 38,98 \\
\hline Transferências a Municípios & $96.246 .041,00$ & 0,25 & - & 0,00 & 0,00 \\
\hline $\begin{array}{l}\text { Transferências a Municípios - } \\
\text { Fundo a Fundo }\end{array}$ & $16.800 .581 .107,00$ & 43,11 & 5.640.948.966,55 & 50,07 & 33,58 \\
\hline $\begin{array}{l}\text { Transferências a Instituições } \\
\text { Privadas sem Fins Lucrativos }\end{array}$ & $12.000 .000,00$ & 0,03 & $5.000 .000,00$ & 0,04 & 41,67 \\
\hline Transferências ao Exterior & $542.294 .000,00$ & 1,39 & $432.376 .875,00$ & 3,84 & 79,73 \\
\hline Aplicações Diretas & $11.589 .288 .382,00$ & 29,74 & $1.302 .728 .873,03$ & 11,56 & 11,24 \\
\hline Total & $38.967 .509 .368,00$ & & 11.267.161.996,92 & & 28,91 \\
\hline
\end{tabular}

Em reais.

Fonte: Elaborada pelos autores com base em SIOP/ME.

Consoante dados do SIOP/ME, do total previsto, aproximadamente $29 \%$ seriam aplicados pelo próprio MS, devendo a maior parte ser repassada aos governos subnacionais para o combate à enfermidade. No entanto, tomando como medida os valores efetivamente pagos até a data de obtenção dos dados, apenas 38,9\% do montante destinado aos estados foram transferidos, porcentagem que cai para 33,5\% em relação aos municípios. No nível federal, a execução foi de apenas 11,24\%. Os valores são praticamente idênticos se calculados com base nos montantes liquidados.

\subsection{Processo de alocação entre estados e municípios}

A Lei 8080 (1990) determinou a descentralização no que concerne ao dever do Estado, expresso na Constituição Federal, de promover políticas sociais e econômicas para a promoção, proteção e recuperação da saúde. Sendo assim, parte dos recursos federais para financiamento das ações e serviços de saúde é transferida aos demais entes subnacionais como cofinanciamento de suas ações. 
O modelo de enfrentamento da COVID-19 segue a mesma lógica e por isso tem os governos subnacionais como agentes fundamentais para sua operacionalização. Isto está claro na própria alocação de recursos, visto que $68,95 \%$ dos valores foram repassados a estados e municípios, correspondendo a $84,5 \%$ do total executado. Não obstante, a realidade de governos subnacionais é muito distinta, sendo fundamental a análise das regras de alocação adotadas, definidas por meio de portarias pelo MS.

Até meados de junho, para atender às medidas provisórias publicadas, diversas normas foram editadas pelo MS, que definiu regras para apoio ao financiamento das ações de enfrentamento da COVID-19 e criou o Programa de Trabalho 10.122.5018.21C0.6500 - Enfrentamento da Emergência de Saúde Pública de Importância Internacional Decorrente do Coronavírus. Até o momento destacam-se sete portarias, detalhadas na Tabela 2, pelas quais são transferidos recursos para ações de enfrentamento à pandemia, autorizados por meio de medidas provisórias ou lei específica. Os recursos foram enviados dentro do Bloco de Manutenção, identificados por um grupo específico para acompanhamento destes repasses: COVID-19.

A portaria n. 395 (2020) repassou um total de 424,15 milhões de reais provenientes do MS aos Fundos Estaduais de Saúde (FES). Os valores foram distribuídos tendo como base o número de habitantes de cada estado e, como regra, R $\$ 2,00$ per capita. O FNS realizou o repasse total aos FES, determinando que a distribuição do recurso no âmbito intraestadual deveria ser pactuada por meio das Comissões Intergestores Bipartite (CIB), considerando o respectivo Plano de Contingência. Não obstante, somente os estados de Pernambuco, Paraná, Rio de Janeiro e Santa Catarina repassaram parte ou o valor total da verba do ministério aos municípios. Assim, a maior parte desses recursos permaneceu nos cofres estaduais.

Em 23 de março de 2020, o MS publicou a Portaria n. 480, destinando R $\$ 600$ milhões aos estados e municípios. Ao contrário, contudo, do documento anterior, o MS definiu um novo método de distribuição. Novamente a pactuação deveria ser por meio da CIB, observando-se, porém, sempre um mínimo de R \$2,00 per capita, de acordo com as estimativas de população do IBGE de 2018 para repasse aos municípios. Uma importante inovação foi garantir o repasse de recursos para prefeituras.

Assim, a portaria definiu que os estados que já repassaram recursos oriundos da Portaria n. 395 (2020) aos municípios poderiam recebê-los desde que pactuados em CIB. Por sua vez, os governos estaduais que não tinham ainda transferido valores aos municípios deveriam desta vez, obrigatoriamente, fazê-lo, tendo sua respectiva alocação pactuada em CIB, além de priorizar as redes assistenciais com maior potencial para enfrentamento da COVID-19. Foi estabelecido, ainda, um valor entre $\mathrm{R}$ \$2,00 a R \$ 5,00 per capita para distribuição aos municípios. O MS determinou um prazo de 24 horas para formalização da distribuição interestadual dos recursos, que seriam então utilizados pelo FNS para o repasse. Neste segundo repasse, $68,29 \%$ do auxílio provindo do MS foram destinados aos municípios.

Já na terceira portaria publicada, n. 774 (2020), a metodologia de distribuição foi alterada. Por meio desta norma foram destinados 3.944.360.945,06 bilhões de reais às demais esferas de governo, utilizando como critério o repasse de uma parcela extra do Piso de Atenção Básica (PAB) para um conjunto de municípios, e para outro grupo de municípios a parcela referente a 1/12 (um doze avos) do Limite Financeiro anual do Grupo de Média e Alta Complexidade Ambulatorial e Hospitalar, dependendo da sua gestão local, desconsiderando, desta forma, as desigualdades regionais e vazios assistenciais existentes. Do total da verba transferida, $66,9 \%$ da quantia foram destinados aos munícipios. 
Mais recentemente, a edição da portaria 827, publicada em 15 de abril de 2020, incluiu o procedimento de complementação de valor de sessão de hemodiálise, bem como seu financiamento, em pacientes com suspeição ou confirmação da COVID-19 na Tabela de Procedimentos, Medicamentos, Órteses, Próteses e Materiais Especiais do SUS. O pagamento é feito por uma Autorização de Procedimento Ambulatorial (APAC) e visa ao atendimento da determinação da ANVISA referente ao uso único das linhas de diálise e dialisadores, por sessão, em pacientes suspeitos ou confirmados de infecção pelo novo coronavírus.

A portaria n. 430, de 19 de março de 2020, estabeleceu incentivo financeiro federal de custeio no âmbito da Atenção Primária à Saúde, de março a setembro de 2020, para apoiar o funcionamento em horário estendido das Unidades de Saúde da Família (USF) ou Unidades Básicas de Saúde (UBS) no país.

Já a portaria n. 568 (2020) é a base para abertura das habilitações de leitos de Unidade de Terapia Intensiva Adulto e Pediátrica para atendimento exclusivo dos pacientes com a COVID-19. Os critérios para habilitação abrangeram dados epidemiológicos (paciente $\mathrm{x}$ leitos) e rede assistencial disponível nos estados. A partir da data de edição desta portaria, foram habilitados 8590 leitos, detalhados por estado na Quadro 1.

QUADRO 1 DISTRIBUIÇÃO DE LEITOS HABILITADOS EM UTI PARA COVID-19 ATÉ 12 DE JUNHO DE 2020

\begin{tabular}{|c|c|c|c|c|c|c|}
\hline \multirow[b]{2}{*}{ UF } & \multicolumn{2}{|c|}{ ESTADUAL } & \multicolumn{2}{|c|}{ MUNICIPAL } & \multirow{2}{*}{$\begin{array}{c}\text { Total Geral } \\
\text { de Leitos }\end{array}$} & \multirow[b]{2}{*}{ VALOR habilitado } \\
\hline & $\begin{array}{c}\text { UTI } \\
\text { ADULTO }\end{array}$ & $\begin{array}{c}\text { UTI } \\
\text { PEDIÁTRICA }\end{array}$ & $\begin{array}{c}\text { UTI } \\
\text { ADULTO }\end{array}$ & $\begin{array}{c}\text { UTI } \\
\text { PEDIÁTRICA }\end{array}$ & & \\
\hline AC & 30 & & & & 30 & $4.320 .000,00$ \\
\hline AL & 61 & & 93 & & 154 & $22.176 .000,00$ \\
\hline AM & 186 & 8 & & & 194 & $27.936 .000,00$ \\
\hline AP & 32 & & & & 32 & $4.608 .000,00$ \\
\hline BA & 111 & & 147 & 17 & 275 & $39.600 .000,00$ \\
\hline CE & 185 & 6 & 186 & 15 & 392 & $56.448 .000,00$ \\
\hline DF & 175 & 10 & & & 185 & $26.640 .000,00$ \\
\hline ES & 88 & & 77 & & 165 & $23.760 .000,00$ \\
\hline GO & 20 & & 139 & & 159 & $22.896 .000,00$ \\
\hline MA & 149 & & 89 & & 238 & $34.272 .000,00$ \\
\hline MG & 38 & & 365 & & 403 & $58.032 .000,00$ \\
\hline MS & 15 & & 152 & & 167 & $24.048 .000,00$ \\
\hline MT & 114 & 10 & 124 & 25 & 273 & $39.312 .000,00$ \\
\hline PA & 270 & 14 & 52 & & 336 & $48.384 .000,00$ \\
\hline PB & 84 & & 80 & 6 & 170 & $24.480 .000,00$ \\
\hline PE & 535 & 15 & 84 & 10 & 644 & $92.736 .000,00$ \\
\hline PI & 10 & & 241 & 10 & 261 & $37.584 .000,00$ \\
\hline PR & 141 & & 297 & 35 & 473 & $68.112 .000,00$ \\
\hline RJ & 50 & & 585 & 16 & 651 & $93.744 .000,00$ \\
\hline $\mathbf{R N}$ & 102 & 10 & 44 & & 156 & $22.464 .000,00$ \\
\hline RO & 53 & 7 & 16 & & 76 & $10.944 .000,00$ \\
\hline RS & 215 & & 404 & 5 & 624 & $89.856 .000,00$ \\
\hline SC & 162 & & 190 & 20 & 372 & $53.568 .000,00$ \\
\hline SE & 40 & & 14 & & 54 & $7.776 .000,00$ \\
\hline SP & 718 & & 1.342 & 4 & 2064 & $297.216 .000,00$ \\
\hline TO & 36 & 6 & & & 42 & $6.048 .000,00$ \\
\hline Total Geral & 3.620 & 86 & 4.721 & 163 & 8590 & $1.236 .960 .000,00$ \\
\hline
\end{tabular}

$\mathrm{R} \$$ em milhões.

Fonte: Elaborada pelos autores com base em Imprensa Nacional. 
Em maio de 2020 foi sancionada a Lei 13.995/20, destinando, por meio dos fundos estaduais e municipais, $\mathrm{R} \$ 2$ bilhões como auxílio financeiro às Santas Casas e aos hospitais filantrópicos, sem fins lucrativos, com o objetivo de ajudá-los a atuar de forma coordenada no combate à COVID-19. A distribuição dos recursos foi realizada em duas parcelas, por meio das portarias 1393 e 1448, publicadas em 21 e 29 de maio de 2020, respectivamente, e teve como base o quantitativo de leitos do SUS do CNES até a data de 12 de maio de 2020, das Santas Casas e dos hospitais filantrópicos constantes nos Planos de Contingências dos estados e Distrito Federal, e dos situados nos municípios que possuem presídios, atribuindo proporcionalmente ao número de leitos de cada estabelecimento o valor da parcela definido.

Os demais repasses, até o momento, foram destinados às propostas aprovadas por emendas parlamentares, tendo como critério a destinação do parlamentar e as propostas de financiamento apresentadas por estados e municípios no Sistema de Gerenciamento de Objetos e Propostas do FNS.

\section{QUADRO 2 DISTRIBUIÇÃO DOS RECURSOS DE EMENDA PARLAMENTAR PARA APOIO À COVID-19 ATÉ 12.06}

\begin{tabular}{|c|c|c|c|c|}
\hline UF & $\begin{array}{l}\text { Valor Pago FAF } \\
\text { Estadual }\end{array}$ & $\begin{array}{l}\text { Quantidade de Munic./UF } \\
\text { recebedores de emenda } \\
\text { enfrentamento covid-19 }\end{array}$ & $\begin{array}{l}\text { Valor Repasse } \\
\text { Municipal por UF }\end{array}$ & Valor total por UF \\
\hline AC & $28.075 .527,00$ & - & & $28.075 .527,00$ \\
\hline$A L$ & $3.500 .000,00$ & 60 & $70.244 .211,00$ & $73.744 .211,00$ \\
\hline AM & $6.500 .000,00$ & 54 & $110.334 .308,00$ & $116.834 .308,00$ \\
\hline AP & $35.457 .173,00$ & 14 & $23.774 .304,00$ & $59.231 .477,00$ \\
\hline BA & $54.869 .588,00$ & 138 & $72.035 .310,00$ & $126.904 .898,00$ \\
\hline CE & $105.970 .421,00$ & 135 & $74.995 .919,00$ & $180.966 .340,00$ \\
\hline DF & $50.437 .829,00$ & - & & $50.437 .829,00$ \\
\hline GO & $18.607 .688,00$ & 158 & $58.195 .820,00$ & $76.803 .508,00$ \\
\hline MA & $43.336 .600,00$ & 95 & $82.239 .642,00$ & $125.576 .242,00$ \\
\hline PB & $4.800 .000,00$ & 21 & $10.800 .000,00$ & $15.600 .000,00$ \\
\hline PE & $54.921 .718,00$ & 31 & $30.159 .782,00$ & $85.081 .500,00$ \\
\hline RJ & & 3 & $97.697 .820,00$ & $97.697 .820,00$ \\
\hline RN & $25.316 .244,00$ & 22 & $13.694 .130,00$ & $39.010 .374,00$ \\
\hline RO & $30.991 .725,00$ & - & & $30.991 .725,00$ \\
\hline RR & $27.000 .000,00$ & 8 & $22.014 .018,00$ & $49.014 .018,00$ \\
\hline RS & $48.518 .312,00$ & 37 & $15.349 .225,00$ & $63.867 .537,00$ \\
\hline SE & $38.650 .461,00$ & 2 & $7.534 .650,00$ & $46.185 .111,00$ \\
\hline SP & $152.861 .216,00$ & 23 & $61.438 .417,00$ & $214.299 .633,00$ \\
\hline TO & - & 2 & $2.000 .000,00$ & $2.000 .000,00$ \\
\hline Total Geral & $679.376 .673,00$ & 804 & $802.945 .385,00$ & $1.482 .322 .058,00$ \\
\hline
\end{tabular}

R\$ em milhões.

Fonte: Elaborada pelos autores com base em FNS (2020).

\subsection{Análise dos Recursos Recebidos}

A análise da distribuição das transferências das portarias publicadas mostra que na esfera estadual a divisão dos recursos seguiu diversos critérios, destacando-se a dimensão populacional, a presença de rede instalada e também fatores políticos, evidenciados nas emendas parlamentares. A Tabela 2 sintetiza os repasses aos governos estaduais e municipais para o enfrentamento da COVID-19. 
RAP | Os desafios do financiamento do enfrentamento à COVID-19 no SUS dentro do pacto federativo

\section{TABELA 2 DISTRIBUIÇÃO DOS RECURSOS POR NORMA PUBLICADA - R\$ EM MILHÕES}

\begin{tabular}{|c|c|c|c|}
\hline Forma de Distribuição de recursos & Est. & Mun. & Total \\
\hline Emenda Parlamentar & 729,81 & 752,51 & $1.482,32$ \\
\hline Habilitação Leito de UTI para COVID-19 & 486,36 & 586,81 & $1.073,17$ \\
\hline Port. 480 & 186,96 & 413,04 & 600,00 \\
\hline Port. 395 & 424,15 & & 424,15 \\
\hline Port. 430 & 0,41 & 8,33 & 8,73 \\
\hline Port. 774 & $1.305,57$ & $2.638,93$ & $3.944,50$ \\
\hline Port. 827 & 12,30 & 24,43 & 36,72 \\
\hline Port. 1393 e 1448 /2020 & 752,77 & $1.247,23$ & $2.000,00$ \\
\hline Total Geral & $3.898,34$ & $5.671,26$ & $9.569,59$ \\
\hline
\end{tabular}

Fonte: Elaborada pelos autores com base em FNS (2020).

Para inferir o impacto deste desenho institucional, regrediu-se o total de transferência recebida por cada município atualizando-o até 12 de junho de 2020, cujos dados estão disponíveis no FNS/MS, em um conjunto de fatores socioeconômicos e geográficos, por meio do método dos mínimos quadrados ordinários com estimadores robustos (Greene, 2002). Para isto, empregou-se a especificação definida na Equação 1.

\section{TABELA 3 ESTATÍSTICAS DESCRITIVAS}

\begin{tabular}{lcccc}
\hline Variável & N. & Média & Mín. & Máx. \\
\hline Transf. Rec. por Mun. & 5568 & $\mathrm{R} \$ 1.012 .714$ & $\mathrm{R} \$ 1.041$ & $\mathrm{R} \$ 278.761 .609$ \\
Gasto Saúde Per Capita & 5564 & $\mathrm{R} \$ 889$ & $\mathrm{R} \$ 0$ & $\mathrm{R} \$ 5.562$ \\
Densidade Pop. & 5566 & 126 & 4 & 25.977 \\
População & 5501 & 37.167 & 812 & 12.106 .920 \\
PIB Municipal & 5561 & $\mathrm{R} \$ 1.168 .620$ & $\mathrm{R} \$ 12.992$ & $\mathrm{R} \$ 699.288 .352$ \\
Leitos & 5570 & 55,16 & 0 & 15.502 \\
Leitos UTI & 5570 & 3,89 & 0 & 1906 \\
Capital & 5570 & 0,00 & 0 & 1 \\
Mort. Infantil & 5570 & 12,26 & 9,9 & 19,60 \\
Dist Brasília (em Km) & 5507 & 1.076 & 0,75 & 2.868 \\
\hline Dist. Capital (em Km) & 5507 & 253 & 0 & 1.476
\end{tabular}

Fonte: IBGE (2020), FNS (2020), CNESS (2020), e FINBRA (2020). 
A Tabela 3 mostra as principais estatísticas das variáveis utilizadas. Todos os dados foram obtidos do Banco de Dados do Tesouro Nacional, o FINBRA, do FNS, do CNES, do IBGE. A cidade de São Paulo foi o município que recebeu o maior repasse, equivalente a $\mathrm{R} \$ 278.761 .608,69$, seguida de Belo Horizonte, com R $\$ 168.920 .165,01$, e Fortaleza, com R $\$ 111.806 .582,03$. Por sua vez, Quixaba-PE, que recebeu apenas $\mathrm{R} \$ 1.041,43$, Bora-SP, com $\mathrm{R} \$ 2.415,73$, e Engenho Velho-RS, com $\mathrm{R} \$ 2.614,18$, são as três cidades com menos recursos recebidos para combate à COVID-19. Aproximadamente 1443 municípios receberam no máximo $\mathrm{R} \$ 50.000,00$. O gasto per capita mais alto em saúde no Brasil é registrado no município gaúcho de Santa Maria, com R \$ 5.561,87 per capita, e o menor em Alvinópolis, com menos de $\mathrm{R} \$ 1,00$ por habitante. As demais variáveis seguem o padrão de grande heterogeneidade no Brasil.

\section{TABELA 4 RESULTADOS}

\begin{tabular}{|c|c|c|c|c|c|}
\hline & \multicolumn{5}{|c|}{ Transf. Recebidas para Enfrentamento COVID-19 } \\
\hline \multirow[t]{2}{*}{ Log Saúde Hab } & $-0,503$ & $-0,359$ & & & $-0,071$ \\
\hline & {$[-8,893]^{\star \star \star}$} & {$[-9,515]^{\star \star \star}$} & & & {$[-1,967]^{\star}$} \\
\hline \multirow[t]{2}{*}{ Log Leitos UTI } & & 0,551 & & & 0,306 \\
\hline & & {$[29,688]^{\star \star \star}$} & & & {$[14,345]^{\star \star \star}$} \\
\hline \multirow[t]{2}{*}{ Log Leitos } & & 0,496 & & & 0,221 \\
\hline & & {$[52,022]^{\star \star \star}$} & & & {$[21,169]^{\star \star \star}$} \\
\hline \multirow[t]{2}{*}{ Log Dens. Hab. } & & & 0,187 & & $-0,011$ \\
\hline & & & {$[9,352]^{\star \star \star}$} & & {$[-0,796]$} \\
\hline \multirow[t]{2}{*}{ Log. Dist Brasília (em Km) } & & & 0,202 & & $-0,048$ \\
\hline & & & {$[6,252]^{\star \star \star}$} & & {$[-2,415]^{\star}$} \\
\hline \multirow[t]{2}{*}{ Log Dist. Capital (em Km) } & & & $-0,244$ & & 0,015 \\
\hline & & & {$[-7,299]^{\star \star \star}$} & & {$[0,873]$} \\
\hline \multirow[t]{2}{*}{ Log População } & & & & 0,250 & 0,203 \\
\hline & & & & {$[11.930]^{\star \star \star}$} & {$[8,658]^{\star \star \star}$} \\
\hline \multirow[t]{2}{*}{ Log PIB } & & & & 0,787 & 0,497 \\
\hline & & & & {$[42.980]^{\star \star \star}$} & {$[25,331]^{\star \star *}$} \\
\hline \multirow[t]{2}{*}{ Log Mort. Infantil } & & & & 3,118 & 2,509 \\
\hline & & & & {$[30.660]^{\star \star \star}$} & {$[21,837]^{\star \star *}$} \\
\hline \multirow[t]{2}{*}{ Capital } & & & & 0,440 & $-0,589$ \\
\hline & & & & {$[1.630]^{*}$} & {$[-2,322]^{\star}$} \\
\hline \multirow[t]{2}{*}{ Constante } & 15.248 & 13.024 & 11.156 & $-7,969$ & -2.233 \\
\hline & {$[40,373]^{\star \star \star}$} & {$[50,727]^{\star \star \star}$} & {$[52,994]^{\star \star *}$} & {$[-26,360]^{\star \star \star}$} & {$[-4,409]^{\star \star \star}$} \\
\hline $\mathrm{R}^{2}$ & 0,014 & 0,559 & 0,064 & 0,629 & 0,675 \\
\hline Observações & 5563 & 5563 & 5559 & 5495 & 5485 \\
\hline
\end{tabular}

Obs. 1: t-valores nos parênteses.

Obs. 2: \# $\mathrm{p}<0.1 ;^{\star} \mathrm{p}<0.05 ;{ }^{* *} \mathrm{p}<0.01 ;{ }^{* *} \mathrm{p}<0$.

Fonte: Elaborada pelos autores. 
A Tabela 4 apresenta os resultados. O primeiro modelo mostra que os municípios com menores gastos per capita em saúde foram contemplados com repasses maiores. No último modelo, com todos os controles, para cada $1 \%$ de queda no gasto per capita em saúde, o município recebeu $0,071 \%$ a mais de repasses. Todavia os valores absolutos médios repassados foram relativamente pequenos.

No entanto, quando se leva em conta o número de leitos e de unidades de tratamento intensivo, infere-se forte correlação positiva. De fato, a habilitação de leitos foi uma importante forma de repartição de recursos e isso é claramente captado no segundo modelo. Este resultado permanece quando são adicionados todos os controles. Ou seja, dentro da estrutura preexistente, concentraram-se os repasses justamente nos locais em que há hospitais com leitos, especialmente, de UTI. Desse modo, vale lembrar que a portaria n. 568 (2020) teve como critério de repasse a abertura das habilitações de leitos de Unidade de Terapia Intensiva Adulto e Pediátrica.

Municípios com maior densidade demográfica, ou mais próximos de Brasília e das respectivas capitais estaduais, não foram contemplados com mais recursos. Apesar do terceiro modelo indicar este resultado, ao se introduzirem mais controles rejeita-se a significância estatística. Por conseguinte, variáveis epidemiológicas com base nas características da doença, que potencialmente visassem conter a expansão da doença, por meio de prevenção, foram preteridas por indicadores relacionados ao aumento da capacidade de tratamento da COVID-19 (Anderson et al., 2020; Feng et al., 2020; Grasselli et al., 2020; Wang et al., 2020).

Por seu turno, municípios com maior população foram beneficiados com maiores transferências. Em especial, o peso da produção de riquezas municipais mostrou-se relevante, sendo estatisticamente significante em todos os modelos: municípios mais ricos receberam maiores valores. É importante destacar que não foi incorporada desigualdade no modelo, de sorte que cidades mais ricas podem ser desiguais, possuindo uma parcela grande de população vulnerável. Nesta direção, aponta a mortalidade infantil, visto que localidades com maiores índices receberam importâncias maiores. Com efeito, para cada $1 \%$ de crescimento da taxa de mortalidade infantil, o município recebeu $2,51 \%$ a mais.

Em suma, os resultados da análise indicam que o modelo de financiamento do SUS não foi modificado em virtude das necessidades de enfrentamento da COVID-19, predominando a lógica de desigualdade preexistente e o viés político (Baptista et al., 2012; Battesini, Andrade, \& Seta, 2017; Piola et al., 2016; Santos 2018).

\section{CONCLUSÃO}

O enfrentamento da COVID-19 é o maior desafio do sistema de saúde brasileiro em décadas. No entanto, em dissonância com as experiências da Itália e da Espanha, manteve-se a situação de subfinanciamento dos serviços de saúde pública. O total de gastos em saúde pública no Brasil cresceu relativamente pouco em termos reais, além de o repasse per capita para a maior parte dos municípios brasileiros ser relativamente pequeno.

Considerando as portarias já publicadas, a habilitação de leitos de UTI e o incentivo destinado à ampliação de horário para atendimento nas USFs, evidenciou-se a priorização dos recursos para Atenção Básica e Especializada. Já as demais portarias de apoio financeiro foram destinadas a quaisquer ações e serviços públicos de saúde para enfrentamento da COVID-19, permitindo maior liberdade de ação aos entes subnacionais, apesar dos impedimentos da categoria de despesa existente. Assim, 
a desvinculação de destinação junto com a recaracterização dos blocos de financiamento ampliou a autonomia da gestão local para atender às demandas existentes.

No tocante à distribuição dos recursos, a coordenação e a forma de alocação ainda demandam aprimoramento. Até o momento, a maior parcela de distribuição de recursos foi por meio do apoio financeiro previsto na portaria n. 774 (2020), com distorções na alocação dos municípios, tendo em vista os critérios estabelecidos. Também foram muito relevantes as emendas parlamentares, muito embora uma parte considerável de municípios não tenha recebido recursos por critérios eminentemente políticos. Em síntese, a lógica de financiamento causou forte desigualdade de destinação dos recursos para apoio ao combate à COVID-19. A habilitação de leitos de UTI ampliou a capacidade instalada nas regiões; contudo, considerando a evolução dos casos em alguns locais, estes leitos podem não atender à totalidade da demanda.

Um importante limitador desta pesquisa é o ineditismo da pandemia. São raros os estudos voltados à apreensão das necessidades de financiamento dos serviços de saúde quando há expressivo aumento da demanda. Além disso, a doença ainda é um fenômeno novo, cujos efeitos devem ser sentidos por muito tempo no SUS. Desse modo, é preciso que novos estudos sejam feitos, avaliando as medidas tomadas relativamente aos dados que ainda serão coletados.

Não obstante, no caso brasileiro, o novo se encontra com o velho, conforme a recorrência de questões já anotadas na literatura sobre financiamento do SUS, dentro do pacto federativo. Em síntese, o modelo de financiamento do SUS não foi modificado diante das necessidades de enfrentamento da COVID-19. Repetem-se, portanto, a lógica de desigualdade preexistente e o viés político, conforme já documentado na literatura (Baptista et al., 2012; Battesini, Andrade, \& Seta, 2017; Piola et al., 2016; Santos, 2018). Trata-se de um importante resultado, pois, a despeito do tamanho da crise imposta pela pandemia, não houve mudança qualitativa no desenho das regras de financiamento, o que intensificou também os problemas de subfinanciamento.

É verdade que houve ajustes nas regras de aplicação nos blocos de financiamento para permitir mais agilidade na adequação da estrutura de enfrentamento e liberação de recursos. No entanto, comparativamente às medidas tomadas por outros países, fortemente coordenadas, as ações no Brasil parecem ser insuficientes diante do desafio enfrentado por sua sociedade. 


\section{REFERÊNCIAS}

Anderson, R. M., Heesterbeek, H., Klinkenberg, D., \& Hollingsworth, T. D. (2020). How will countrybased mitigation measures influence the course of the COVID-19 epidemic? The Lancet, 395(10228), 931-934. Recuperado de https://doi.org/10.1016/ S0140-6736(20)30567-5

Araújo, C. E. L., Gonçalves, G. Q., \& Machado, J. A. (2017). Os municípios brasileiros e os gastos próprios com saúde: algumas associações. Ciência \& Saúde Coletiva, 22(3), 953-963. Recuperado de https://doi. org/10.1590/1413-81232017223.15542016

Araújo, I. G., \& Morais, A. C. L. N. (2020). Cenário atual da covid-19 no estado do Ceará, Brasil. InterAmerican Journal of Medicine and Health, 3, 1-9. Recuperado de https://doi. org/10.31005/iajmh.v3i0.92

Banco de Dados do Tesouro Nacional. (2020). FINBRA. Brasília, DF: Autor.

Baptista, T. W. F., Machado, C. V., Lima, L. D., Garcia, M., Andrade, C. L. T., \& Gerassi, C. D. (2012). As emendas parlamentares no orçamento federal da saúde. Cadernos de Saúde Pública, 28(12), $2267-$ 2279. Recuperado de https://doi.org/10.1590/S0102311X2012001400006

Battesini, M., Andrade, C. L. T., \& Seta, M. H. (2017). Financiamento federal da Vigilância Sanitária no Brasil de 2005 a 2012: análise da distribuição dos recursos. Ciência \& Saúde Coletiva, 22(10), 3295-3306. Recuperado de https://dx.doi. org/10.1590/1413-812320172210.10852017

Cabreira, F. S., Ritter, F., Aguiar, V. R., \& Celeste, R. K. (2018). Despesas municipais em atenção primária à saúde no Rio Grande do Sul, Brasil: um estudo ecológico. Cadernos de Saúde Pública, 34(12), e00150117. Recuperado de https:// doi.org/10.1590/0102-311x00150117

Cadastro Nacional de Estabelecimentos de Saúde. (2020). CNES. Brasília, DF: Autor.

Conselho Nacional de Secretarias Municipais de Saúde. (2020). Nota Técnica - perguntas e respostas live - Normas sobre o financiamento do SUS estabelecidas em decorrência do COVID-19. Recuperado de https://www.conasems.org.br/ wp-content/uploads/2020/04/PDF-PERGUNTASRESPOSTAS.pdf
Contarato, P. C., Lima, L. D., \& Leal, R. M. (2019). Crise e federalismo: tendências e padrões regionais das receitas e despesas em saúde dos estados brasileiros. Ciência \& Saúde Coletiva, 24(12), 44154426. Recuperado de https://doi.org/10.1590/1413812320182412.25302019

Chan, J. F. W., Yuan, S., Kok, K. H., To, K. K. W., Chu, H., Yang, J., ... Tsoi, H. W. (2020). A familial cluster of pneumonia associated with the 2019 novel coronavirus indicating person-to-person transmission: a study of a family cluster. The Lancet, 395(10223), 514-523. Recuperado de https:// doi.org/10.1016/S0140-6736(20)30154-9

Emenda constitucional n. 95, de 15 de dezembro de 2016. (2016). Altera o Ato das Disposições Constitucionais Transitórias, para instituir o Novo Regime Fiscal, e dá outras providências. Brasília, DF. Recuperado de http://www.planalto.gov.br/ ccivil_03/constituicao/emendas/emc/emc95.htm

Feng, S., Shen, C., Xia, N., Song, W., Fan, M., \& Cowling, B. J. (2020). Rational use of face masks in the COVID-19 pandemic. The Lancet Respiratory Medicine, 8(5), 434-436. Recuperado de https://doi. org/10.1016/S2213-2600(20)30134-X

Funcia, F. R. (2019). Subfinanciamento e orçamento federal do SUS: referências preliminares para a alocação adicional de recursos. Ciência \& Saúde Coletiva, 24(12), 4405-4415. Recuperado de https:// dx.doi.org/10.1590/1413-812320182412.25892019

Fundo Nacional da Saúde. (2020). FNE. Brasília, DF: Autor.

Grasselli, G., Pesenti, A., \& Cecconi, M. (2020). Critical care utilization for the COVID-19 outbreak in Lombardy, Italy: early experience and forecast during an emergency response. Jama, 323(16), 1545-1546. Recuperado de https://doi:10.1001/ jama.2020.4031

Greene, W. H. (2000). Econometric analysis (4th edition, International edition, pp. 201-215). New Jersey, NY: Prentice Hall.

Harzheim, E., D’Avila, O. P., Ribeiro, D. C., Ramos, L. G., Silva, L. E., Santos ... Pedebos, L. A. (2020). Novo financiamento para uma nova Atenção Primária à Saúde no Brasil. Ciência \& Saúde Coletiva, 25(4), 1361-1374. Recuperado de https:// doi.org/10.1590/1413-81232020254.35062019 
Instituto Brasileiro de Geografia e Estatística. (2020). IBGE. Rio de Janeiro, RJ: Autor.

Kilsztajn, S., Rossbach, A., Câmara, M. B., \& Carmo, M. S. N. (2003). Serviços de saúde, gastos e envelhecimento da população brasileira. Revista Brasileira de Estudos de População, 20(1), 93-108.

Legido-Quigley, H., Mateos-García, J. T., Campos, V. R., Gea-Sánchez, M., Muntaner, C., \& McKee, M. (2020). The resilience of the Spanish health system against the COVID-19 pandemic. The Lancet Public Health, 5(5), e251-e252. Recuperado de https://doi. org/10.1016/S2468-2667(20)30060-8

Lei n. 13.995, de 5 de maio de 2020. (2020). Dispõe sobre a prestação de auxílio financeiro pela União às santas casas e hospitais filantrópicos, sem fins lucrativos, que participam de forma complementar do Sistema Único de Saúde (SUS), no exercício de 2020, com o objetivo de permitir-lhes atuar de forma coordenada no combate à pandemia da Covid-19. Brasília, DF. Recuperado de http://www. planalto.gov.br/CCIVIL_03/_Ato2019-2022/2020/ Lei/L13995.htm

Leite, V. R., Lima, K. C., \& Vasconcelos, C. M. (2012). Financiamento, gasto público e gestão dos recursos em saúde: o cenário de um estado brasileiro. Ciência \& Saúde Coletiva, 17(7), 1849-1856. Recuperado de https://doi.org/10.1590/S1413-81232012000700024

Lima, L. D. (2007). Conexões entre o federalismo fiscal e o financiamento da política de saúde no Brasil. Ciência \& Saúde Coletiva, 12(2), 511 522. Recuperado de https://doi.org/10.1590/S141381232007000200027

Lima, L. D., \& Andrade, C. L. T. (2009). Condições de financiamento em saúde nos grandes municípios do Brasil. Cadernos de Saúde Pública, 25(10), 22372248. Recuperado de https://doi.org/10.1590/S0102311X2009001000014

Marques, R. M. (2017, julho-dezembro). Notas exploratórias sobre as razões do subfinanciamento estrutural do SUS. Planejamento e Políticas Públicas, (49), 35-53.

McNabb, D. E. (2002). Research methods in public administration and nonprofit management. Armonk, New York, NY; London, UK: ME Sharpe.

Medida provisória no 924, de 13 de março de 2020. (2020). Abre crédito extraordinário, em favor dos
Ministérios da Educação e da Saúde, no valor de $\mathrm{R} \$$ 5.099.795.979,00, para os fins que especifica. Recuperado de http://www.in.gov.br/en/web/ dou/-/medida-provisoria-n-924-de-13-de-marcode-2020-247887516

Medida Provisória n 940, de 2 de abril de 2020. (2020). Abre crédito extraordinário, em favor do Ministério da Saúde, no valor de R\$9.444.373.172,00, para os fins que especifica. Recuperado de http://www. planalto.gov.br/CCIVIL_03/_Ato2019-2022/2020/ $\mathrm{Mpv} / \mathrm{mpv} 940 . \mathrm{htm}$

Medida Provisória no 941, de 2 de abril de 2020. (2020). Abre crédito extraordinário, em favor dos Ministérios da Educação, da Saúde e da Cidadania, no valor de $\mathrm{R} \$ 2.113 .789 .466,00$, para os fins que especifica. Recuperado de https://www.planalto.gov. br/ccivil_03/_ato2019-2022/2020/mpv/mpv941.htm

Medida Provisória no 947, de 8 de abril de 2020. (2020). Abre crédito extraordinário, em favor do Ministério da Saúde, no valor de R $\$ 2.600 .000 .000,00$, para os fins que especifica. Recuperado de https:// www.planalto.gov.br/ccivil_03/_ato2019-2022/2020/ $\mathrm{mpv} / \mathrm{mpv} 947 . \mathrm{htm}$

Medida Provisória $n^{\circ}$ 967, de 19 de maio de 2020. (2020). Abre crédito extraordinário, em favor do Ministério da Saúde, no valor de R\$ 5.566.379.351,00, para os fins que especifica e dá outras providências. Recuperado de http://www. planalto.gov.br/CCIVIL_03/_Ato2019-2022/2020/ $\mathrm{Mpv} / \mathrm{mpv} 967 . \mathrm{htm}$

Medida Provisória $n^{\circ}$ 969, de 20 de maio de 2020. (2020). Abre crédito extraordinário, em favor do Ministério da Saúde, no valor de R\$ 10.000.000.000,00, para os fins que especifica, e dá outras providências. Recuperado de http://www. planalto.gov.br/CCIVIL_03/_Ato2019-2022/2020/ $\mathrm{Mpv/mpv969.htm}$

Medida Provisória no 976, de 4 de junho de 2020. (2020). Abre crédito extraordinário, em favor do Ministério da Saúde, no valor de R \$4.489.224.000,00, para ofim que especifica. Recuperado de http://www. planalto.gov.br/ccivil_03/_Ato2019-2022/2020/ Mpv/mpv976.htm

Mendes, A. (2014). O fundo público e os impasses do financiamento da saúde universal brasileira. Saúde e Sociedade, 23(4), 1183-1197. Recuperado de https:// doi.org/10.1590/S0104-12902014000400006 
Mendes, A., \& Marques, R. M. (2009). Ofinanciamento do SUS sob os "ventos" da financeirização. Ciência \& Saúde Coletiva, 14(3), 841-850. Recuperado de https://doi.org/10.1590/S1413-81232009000300019

O’Dwyer, G., Graever, L., Britto, F. A., Menezes, T., \& Konder, M. T. (2019). A crise financeira e a saúde: o caso do município do Rio de Janeiro, Brasil. Ciência \& Saúde Coletiva, 24(12), 4555-4568. Epub, November 25, 2019. Recuperado de https://dx.doi. org/10.1590/1413-812320182412.23212019

Portaria n. 355 de 16 de março de 2020. (2020). Estabelece recurso do Bloco de Custeio das Ações e Serviços Públicos de Saúde - Grupo de Atenção de Média e Alta Complexidade-MAC, a ser disponibilizado aos Estados e Distrito Federal, destinados às ações de saúde para o enfrentamento do Coronavírus - COVID 19. Brasília, DF. Recuperado de http://www.in.gov. br/en/web/dou/-/portaria-n-395-de-16-de-marcode-2020-estabelece-recurso-do-bloco-de-custeiodas-acoes-e-servicos-publicos-de-saude-grupode-atencao-de-media-e-alta-complexidade-mac-aser-disponibilizado-aos-estados-e-distrito-federaldestinados-as-acoes-de-saude-para-o-

Portaria n. 480 de 23 de março de 2020. (2020). Estabelece recurso do Bloco de Custeio das Ações e Serviços Públicos de Saúde, a ser disponibilizado aos estados e Distrito Federal, destinados às ações de saúde para o enfrentamento do Coronavírus COVID 19. Brasília, DF. Recuperado de http://www. in.gov.br/en/web/dou/-/portaria-n-480-de-23-demarco-de-2020-249621650

Portaria n. 568 de 26 de março de 2020. (2020). Autoriza a habilitação de leitos de Unidade de Terapia Intensiva Adulto para atendimento exclusivo dos pacientes COVID-19. Brasília, DF. Recuperado de http://www.in.gov.br/en/web/dou/-/portaria-n568-de-26-de-marco-de-2020-249862050

Portaria n. 774 de 9 de abril de 2020. (2020). Estabelece recursos do Bloco de Custeio das Ações e dos Serviços Públicos de Saúde a serem disponibilizados aos Estados, Distrito Federal e Municípios, destinados ao custeio de ações e serviços relacionados à COVID 19. Brasília, DF. Recuperado de http://www.in.gov.br/en/web/dou/-/portaria-n774-de-9-de-abril-de-2020-*-252498248

Portaria n. 828 de 17 de abril de 2020. (2020). Altera a Portaria de Consolidação n 6/2017/GM/
MS, para dispor sobre os Grupos de Identificação Transferências federais de recursos da saúde. Brasília, DF. Recuperado de http://www.in.gov. br/en/web/dou/-/portaria-n-828-de-17-de-abrilde-2020-253755257

Portaria n. 1393 de 21 de maio de 2020. (2020). Dispõe sobre o auxílio financeiro emergencial às santas casas e aos hospitais filantrópicos sem fins lucrativos, que participam de forma complementar do Sistema Único de Saúde (SUS), no exercício de 2020, com o objetivo de permitir-lhes atuar de forma coordenada no controle do avanço da pandemia da Covid-19. Brasília, DF. Recuperado de http://www. in.gov.br/en/web/dou/-/portaria-n-1.393-de-21-demaio-de-2020-258046968

Portaria n. 1448 de 29 de maio de 2020. (2020). Dispõe sobre a transferência da segunda parcela do auxílio financeiro emergencial às santas casas e aos hospitais filantrópicos sem fins lucrativos, nos termos da Lei $\mathrm{n}^{\circ} 13.995$, de 5 de maio de 2020, e do art. $3^{\circ}$ da Portaria $n^{\circ} 1.393 / \mathrm{GM} / \mathrm{MS}$, de 21 de maio de 2020. Brasília, DF. Recuperado de http://www. in.gov.br/en/web/dou/-/portaria-n-1.448-de-29-demaio-de-2020-*-259490693

Pereira, B. L., \& Faleiros, D. (2019). Desvinculação Orçamentária e o Financiamento da Saúde. Recuperado de https://www.conasems.org.br/ wp-content/uploads/2019/04/DesvinculaçãoOrçamentária-Análise-Conasems-1.pdf

Pereira, B. L. S., Oliveira, A. C. R. D., Jr., \& Faleiros, D. R. (2019). Portaria 3992/2017: desafios e avanços para gestão dos recursos no Sistema Único de Saúde. Revista de Saúde Pública, 53, 58. Recuperado de https://doi.org/10.11606/s15188787.2019053001052

Piola, S. F., França, J. R. M., \& Nunes, A. (2016). Os efeitos da Emenda Constitucional 29 na alocação regional dos gastos públicos no Sistema Único de Saúde no Brasil. Ciência \& Saúde Coletiva, 21(2), 411422. Recuperado de https://doi.org/10.1590/141381232015212.10402015

Pinto, V. L., Jr., Cerbino, J., Neto, \& Penna, G. O. (2014). The evolution of the federal funding policies for the public health surveillance component of Brazil's Unified Health System (SUS). Ciência \& Saúde Coletiva, 19(12), 4841-4849. Recuperado de https:// doi.org/10.1590/1413-812320141912.05962013 
Portal da Transparência. (2020). Enfrentamento da emergência de saúde pública de importância internacional decorrente do coronavírus. Recuperado de http://www.portaltransparencia.gov.br/ programas-e-acoes/acao/21C0-enfrentamentoda-emergencia-de-saude-publica-de-importanciainternacional-decorrente-do-coronavirus

Portaria de consolidação $n$. 6 , de 28 de setembro de 2017. (2017). Consolidação das normas sobre o financiamento e a transferência dos recursos federais para as ações e os serviços de saúde do Sistema Único de Saúde. Recuperado de http://svs.aids.gov.br/dantps/cgiae/vigilanciado-obito/servico-verificacao-obito/portariaconsolidacao-6-28092017.pdf

Santos, L. (2018). SUS-30 anos: um balanço incômodo? Ciência \& Saúde Coletiva, 23(6), 20432050. Recuperado de https://doi.org/10.1590/141381232018236.06082018

Scatena, J. H. G., Viana, A. L. A., \& Tanaka, O. Y. (2009). Sustentabilidade financeira e econômica do gasto público em saúde no nível municipal: reflexões a partir de dados de municípios matogrossenses. Cadernos de Saúde Pública, 25(11), 2433-2445. Recuperado de https://doi.org/10.1590/ S0102-311X2009001100013
Teles, A. S., Coelho, T. C. B., \& Ferreira, M. P. S. (2016). Sob o prisma da equidade: financiamento federal do Sistema Único de Saúde no estado da Bahia. Saúde e Sociedade, 25(3), 786-799. Recuperado de https:// doi.org/10.1590/s0104-12902016152020

Vazquez, D. A. (2011). Efeitos da regulação federal sobre o financiamento da saúde. Cadernos de Saúde Pública, 27(6), 1201-1212. Recuperado de https:// doi.org/10.1590/S0102-311X2011000600017

Walker, P., Whittaker, C., Watson, O., Baguelin, M., Ainslie, K., Bhatia, S., ... Cucunuba Perez, Z. (2020, 26 de março). Report 12: The global impact of COVID-19 and strategies for mitigation and suppression. Imperial College COVID-19 Response Team, 1-18. Recuperado de https://doi. org/10.25561/77735

Wang, C., Horby, P. W., Hayden, F. G., \& Gao, G. F. (2020). A novel coronavirus outbreak of global health concern. The Lancet, 395(10223), 470-473. Recuperado de https://doi.org/10.1016/S01406736(20)30185-9

Weiss, P., \& Murdoch, D. R. (2020). Clinical course and mortality risk of severe COVID-19. The Lancet, 395(10229), 1014-1015. Recuperado de https://doi.org/10.1016/S0140-6736(20)30633-4

\section{Gustavo Andrey de Almeida Lopes Fernandes}

https://orcid.org/0000-0001-8281-390X

Doutor em Economia pela Universidade de São Paulo (USP); Professor da Escola de Administração de Empresas de São Paulo da Fundação Getulio Vargas (FGV EAESP). E-mail: gustavo.fernandes@fgv.br

\section{Blenda Leite Saturnino Pereira}

https://orcid.org/0000-0002-7831-0258

Doutoranda em Saúde Pública pela Faculdade de Saúde Pública da Universidade de São Paulo (USP).

E-mail: blendapereira@usp.br 